\title{
Absolute Transverse Mobility and Ratchet Effect on Periodic 2D Symmetric Substrates
}

\author{
C. Reichhardt and C.J. Olson Reichhardt \\ Center for Nonlinear Studies and Theoretical Division, Los Alamos National Laboratory, Los Alamos, New Mexico 87545
}

(June 19, 2018)

\begin{abstract}
We present a simple model of an overdamped particle moving on a two dimensional symmetric periodic substrate with a dc drive in the longitudinal direction and additional ac drives in both the longitudinal and transverse directions. For certain regimes we find that a finite longitudinal dc force produces a net dc response only in the transverse direction, which we term absolute transverse mobility. Additionally we find regimes exhibiting a ratchet effect in the absence of an applied dc drive.
\end{abstract}

PACS numbers: 05.60,-k, 05.45.-a, 74.25.Qt, 87.16.Uv

\section{INTRODUCTION}

When an overdamped particle is driven with a dc drive, it moves in the direction of the drive, and in the absence of any other external forces the particle velocity increases linearly with the drive. If there is some form of pinning from a substrate, then in general for a finite range of low drives the particle will be immobile or pinned [1,2]. For higher drives in the presence of pinning the velocity vs force curves can be highly nonlinear $[1,2]$. For certain asymmetric substrate potentials, the particle speed can decrease with increasing applied drive, an effect that is termed negative differential resistance [3]. Such effects can also occur for collections of classical coupled particles interacting with periodic substrates [4]. In addition a particle may exhibit a finite average dc velocity in the absence of any external dc drive. This is often referred to as a ratchet effect, which can be thermal [5] or deterministic [6]. Typically in ratchet systems there is some form of underlying asymmetric potential which leads to a spatial symmetry breaking if the potential is flashed or if an additional external ac drive is present. In the case of absolute negative mobility, when the particle is driven in the positive direction, its motion is in the opposite (negative) direction. Examples of this occur in ratchet systems composed of coupled particles, where the collective effects produce the negative mobility [7]. More recently a spatially symmetric two-dimensional (2D) system was found which exhibits absolute negative mobility for a single classical particle [8]. Many of these phenomena, such as negative differential resistance and absolute negative mobility, also occur in various semiconductor devices, where they arise due to quantum effects [9].

In a $2 \mathrm{D}$ system, there are additional possibilities for the motion of an overdamped particle which are not available in 1D systems. Under an external dc drive in the longitudinal or $x$ direction, the response can be a finite velocity in the $y$ or transverse direction only. We call such a phenomenon absolute transverse mobility.
In this work we present a simple model for a driven classical overdamped particle moving in a $2 \mathrm{D}$ symmetric potential that exhibits a variety of dynamical behaviors, including phase locking, absolute transverse mobility, and ratchet effects. Additionally we find reentrant pinning phenomena where the moving particle becomes pinned upon increasing the drive. Our system consists of a particle moving over a symmetric periodic potential with an applied dc drive in the $x$-direction and two additional ac drives in both the $x$ and $y$ directions. We set the amplitudes and frequencies for the two ac drives separately, and also consider highly nonlinear combinations of the ac drives which produce asymmetric closed orbits. In all cases, in the absence of a substrate and dc drive the average dc particle velocity is zero. Previous work on a similar system was performed with much simpler circular particle orbits, produced by setting the amplitudes of both ac drives equal to each other and fixing the phase difference at $90^{\circ}[10]$.

In the previous work, several symmetrical phases were observed where the particle moves in both the transverse and longitudinal direction simultaneously when the dc drive is applied only in the longitudinal direction. In the current work we consider the case where the amplitudes or frequencies of the two ac drives are different. Here a far richer variety of classical closed orbits are realizable. The best examples of such orbits are Lissajous figures in which different sinusoidal orbits are plotted against one another.

Our results should apply to vortices moving in superconductors with periodic pinning arrays or Josephson junction arrays [11-15] when ac currents are applied in both the transverse and longitudinal directions. Additionally, the behavior described here should be observable in colloids moving over $2 \mathrm{D}$ periodic light arrays $[16,17]$ or through dynamically manipulated arrays of holographic tweezers [18]. Further systems include biomolecules moving through periodic arrays of obstacles with two applied electric fields [19], electrons in a strong magnetic field 
moving through $2 \mathrm{D}$ antidot arrays with an additional ac drive to elongate one direction of the electron orbit [20], or ions moving in dissipative optical trap arrays [21] with ac applied fields. In the case of superconductors our results can also have applications for the controlled motion or removal of flux from superconductors and SQUIDs. For colloids and biomolecules our results could provide a useful method for separating different particle species.

\section{MODEL}

In our model we consider an overdamped particle moving over a $2 \mathrm{D}$ periodic substrate. The equation of motion is:

$$
\mathbf{f}=\mathbf{f}_{s}+\mathbf{f}_{D C}+\mathbf{f}_{A C}=\eta \frac{d \mathbf{r}}{d t}
$$

with the damping constant $\eta=1$. The assumption of overdamped motion should be valid for vortices in superconductors or Josephson junction arrays as well as for colloidal systems. The substrate consists of a periodic square array of obstacles with lattice constant $a$. The force from the substrate, composed of a fixed square array of repulsive particles, is $\mathbf{f}_{s}=\sum-\nabla U(x, y)$. This substrate can be realized in superconductors with a square periodic array of holes $[11,12]$ or magnetic dots [14] when each site captures one vortex and additional vortices sit in the interstitial regions between the sites. The interstitial vortices move in a periodic potential created by the repulsive interaction from the pinned vortices so that $U(r)=F_{0} \ln (r)$ for $r \ll 2 \lambda^{2} / d$. Here forces are measured in units of $F_{0}=\Phi_{0}^{2} d / 16 \pi \lambda^{2}$, where $\Phi_{0}$ is the flux quantum, $d$ is the film thickness, and $\lambda$ is the London penetration depth. This potential can be treated as in Ref. [22]. The motion of interstitial vortices has been directly imaged in experiments with this geometry [12], and phase-locking effects, dc, and ac driven interstitial vortex motion have also been observed [13]. For most of the results presented here we use a system of size $8 a \times 8 a$. For larger systems we observe the same results, indicating that our system is large enough to capture the essential physics. We have tested different initial conditions by placing the particle at different locations at the start of the simulations and find that the general results are unchanged. Additionally, we have considered other potentials, such as those created by fixed Coulomb charges or Yukawa potentials, and find that they produce the same phases.

Throughout this work the dc drive $\mathbf{f}_{D C}=f_{D C} \hat{\mathbf{x}}$ is applied along the positive longitudinal ( $x$ direction) symmetry axis of the pinning array. The ac drive is applied in both the $x$ and $y$ directions and is given by

$$
\mathbf{f}_{A C}=A \sin \left(\omega_{A} t\right) \hat{\mathbf{x}}-B \cos \left(\omega_{B} t\right) \hat{\mathbf{y}} .
$$

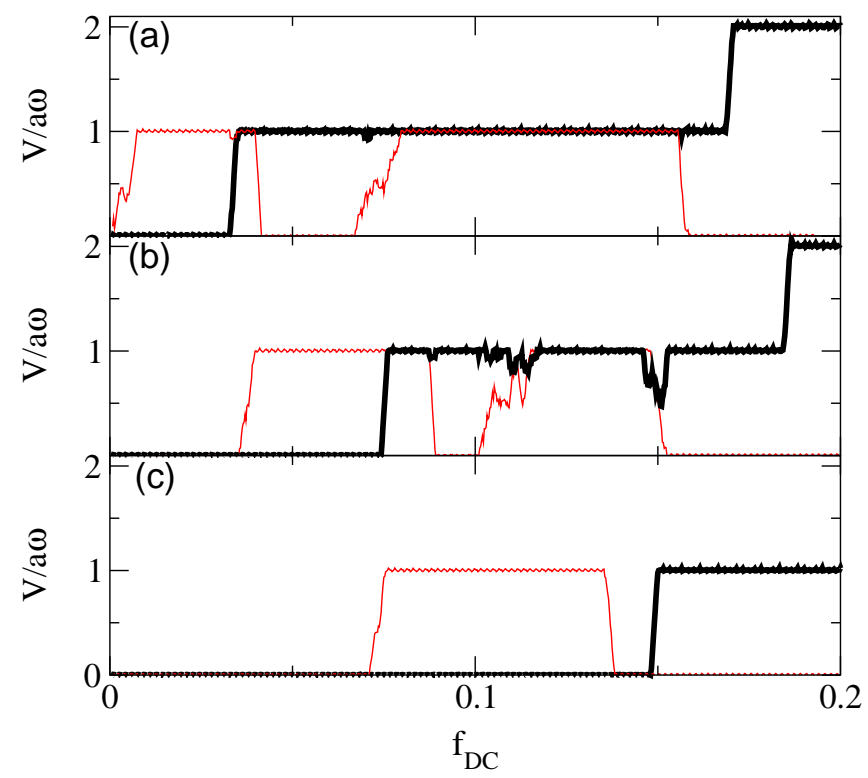

FIG. 1. The longitudinal particle velocity $V_{x} / a \omega$ (heavy lines) and transverse particle velocity $V_{y} / a \omega$ (light lines) vs applied dc drive $\mathbf{f}_{\mathrm{DC}}=f_{D C} \hat{\mathbf{x}}$ for ac amplitudes (a) $A / B=0.875$, (b) $A / B=0.625$, and (c) $A / B=0.375$.

Note that there is no dc driving component in the transverse or $y$ direction. In the first part of this work we set $\omega_{A}=\omega_{B}$, fix $B=0.24$, and vary $A$. For $A=B$ the particle moves in a clockwise circle just large enough to encircle one maximum of the substrate potential. As $A$ decreases the particle orbit becomes elliptical with the long side in the $y$-direction. We monitor the time averaged particle velocity $V_{x}=\frac{1}{T N} \sum_{t=0}^{T} \sum_{i=0}^{N} v_{i}(t) \cdot \hat{\mathbf{x}}$, where $T$ is the period and $N$ is the number of particles, and similarly the time averaged transverse velocity $V_{y}$ as $\mathbf{f}_{D C}$ is increased from 0 to 1.0 in increments of $2.5 \times 10^{-4}$, with $3 \times 10^{5}$ time steps spent at each drive to ensure a steady state. Time is measured in units of $t_{0}=\eta / F_{0}$. We also investigate $\omega_{B} \neq \omega_{A}$ for varied ac amplitude. In this case we find a ratchet effect.

\section{ABSOLUTE TRANSVERSE MOBILITY}

In Fig. 1 we plot $V_{x}$ (heavy line) and $V_{y}$ (light line) vs $f_{D C}$ for different values of $A / B$ at fixed $\omega_{A} / \omega_{B}=1$ and $a=1.42 \lambda$. In Fig. 1 (a) at $A / B=0.875, V_{x}=0$ for $f_{D C}<0.03$, indicating that the particle is pinned in the $x$-direction. For $f_{D C} \geq 0.03, V_{x}$ increases in a series of steps of height $a \omega$. These steps are a signature of the phase locking which occurs due to resonances between the applied ac frequency and the washboard frequency generated as the particle moves over the periodic substrate. As shown in Fig. 1(a), $V_{y}$ has a finite value of $V_{y}=a \omega$ for $f_{D C}<0.03$, indicating that even though the dc drive is strictly in the $x$-direction the particle is moving strictly in the $y$-direction. In analogy with the 


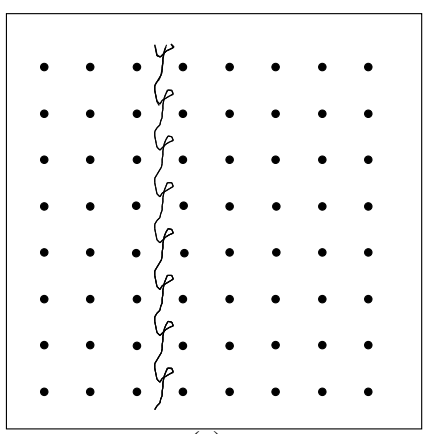

(a)

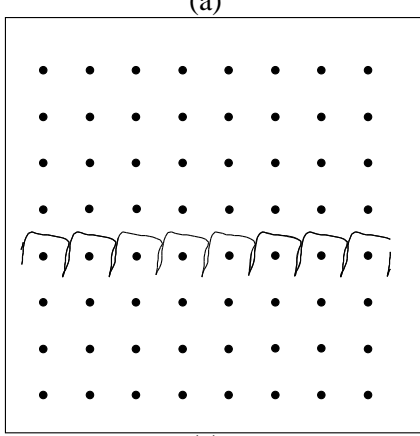

(c)

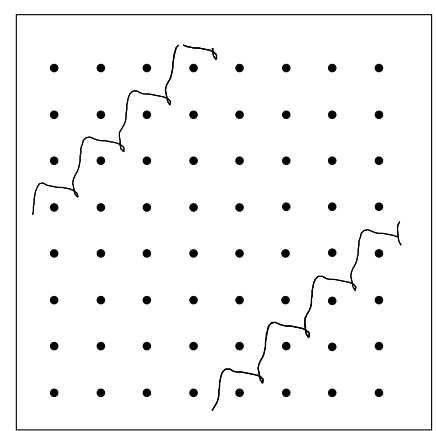

(b)

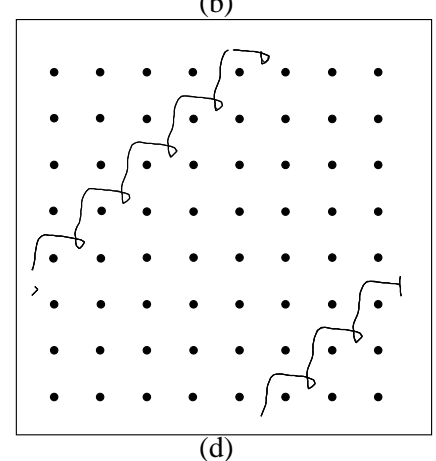

(d)

FIG. 2. The particle trajectories (black lines) for a fixed time interval for the system in Fig. 1(a) with $A / B=0.875$. The black dots are the potential maxima from the periodic substrate. (a) $f_{D C}=0.025$, Phase $\mathrm{II}_{y}$; (b) $f_{D C}=0.0375$, Phase $\mathrm{III}_{x-y} ; \quad$ (c) $f_{D C}=0.06$, Phase $\mathrm{IV}_{x} ;$ and (d) $f_{D C}=0.125$, Phase $\operatorname{III}_{x-y}$.

phenomena of absolute negative mobility, where a particle moves in the opposite direction of an applied driving force, we term the strictly $y$ direction motion absolute transverse mobility. For large enough drives, $f_{D C} \geq 0.16$, the motion is strictly in the $x$ direction. At intermediate drives $0.03<f_{D C}<0.16$, different dynamical phases appear. For $0.045<f_{D C}<0.065$ the particle moves in the $x$-direction only, while for $0.07<f_{D C}<0.158$, $V_{x}=V_{y}$, indicating that the particle is moving at $45^{\circ}$ with respect to the drive. There is also a small region near $f_{D C}=0.035$ where $45^{\circ}$ motion occurs. In Fig. 1(b) for $A / B=0.625$ there is a clear pinned phase at low $f_{D C}$ where both $V_{x}$ and $V_{y}$ are zero. As we increase the drive, we observe the same phases shown in Fig. 1(a), with the boundaries shifted. Near the transitions of these phases, smaller steps in $V_{x}$ and $V_{y}$ can occur with height paw/q where $p$ and $q$ are integers. In Fig. 1(c), for $A / B=0.375$, $45^{\circ}$ motion no longer appears. Instead, there is a remarkable reentrant pinned phase for $0.1385<f_{D C}<0.148$. As $f_{D C}$ increases, the particle is first pinned, then moves in the $y$-direction, is repinned again, and finally moves in the $x$-direction.

We next consider the particle trajectories in these different phases. We term the pinned regime Phase $\mathrm{I}_{p}$, the absolute transverse mobility regime $\mathrm{Phase} \mathrm{II}_{y}$, the $45^{\circ}$ motion regime Phase $\mathrm{III}_{x-y}$, and the strictly $x$ direction motion Phase $\mathrm{IV}_{x}$. In Fig. 2 we illustrate these phases

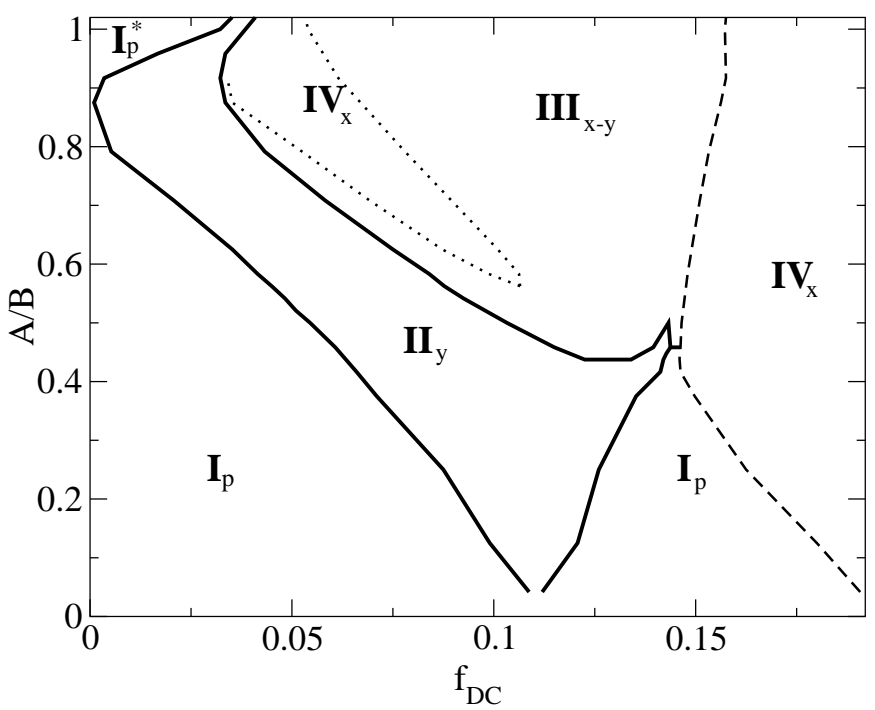

FIG. 3. Dynamic phase diagram for $A / B$ vs $f_{D C}$ for a system with $\omega_{A}=\omega_{B}$. Phase $\mathrm{I}_{p}$ : pinned phase. Phase $\mathrm{II}_{y}$ : motion only in the $y$-direction. Phase $\mathrm{III}_{x-y}$ : motion at $45^{\circ}$. Phase $\mathrm{IV}_{x}$ : motion only in the $x$-direction.

for fixed values of $f_{D C}$ from the system in Fig. 1(a) with $A / B=0.875$. The black lines are the trajectories of the moving particle and the black dots are the potential maxima of the underlying periodic substrate. Figure 2(a) shows the trajectory of the moving particle in Phase $\mathrm{II}_{y}$ at $f_{D C}=0.025$. In every period the particle makes a small loop, but the net motion of the particle is in the positive $y$ direction only. In Phase $\mathrm{III}_{x-y}$, shown in Fig. 2(b) for $f_{D C}=0.0375$, the particle moves equal distances in the $x$ and $y$ directions. At $f_{D C}=0.06$, illustrated in Fig. 2(c), the Phase $\mathrm{IV}_{x}$ motion is strictly in the $x$ direction, and the particle translates a distance $a$ every period. In Fig. 2(d) we show the reentrant Phase III $_{x-y}$ flow for $f_{D C}=0.125$, very similar to that seen in Fig. 2(b). For higher drives $f_{D C}>0.16$, the motion is strictly in the $x$ direction and is similar to Fig. 2(c).

For the simulations shown in Figs. 1 and 2, the particle initially begins in the center of the plaquette with zero dc drive. We considered the effect both of using different starting locations, and of setting $f_{D C}$ to a finite initial value. In each case we find that the particle quickly settles into a regular orbit. The orbits that appear when $V_{x}$ or $V_{y}$ are constant correspond to the same periodic, phase locked attractor orbits that were obtained previously in Figs. 1 and 2. We do find some non-periodic orbits in the regions where $V_{x}$ or $V_{y}$ are not constant, such as in the transition regimes between the different phases.

We performed a series of simulations at different values of $A / B$ to identify the onset of the four phases as a function of $f_{D C}$. In Fig. 3 we present the resulting dynamic phase diagram $A / B$ vs $f_{D C}$ which shows a very rich structure. For $A / B=0$ the system depins directly into Phase $\mathrm{IV}_{x}$ and there are no phases that involve motion in the $y$-direction. Phase $\mathrm{II}_{y}$ first occurs for $A / B>0.03$, 
and gradually increases in width until $A / B \approx 0.43$, when Phase III $_{x-y}$ appears. The reentrant pinning Phase $\mathrm{I}_{p}$ decreases in size and then disappears over this same interval. There is also a small region around $A / B \gtrsim 0.43$ where Phase $\mathrm{II}_{y}$ and Phase $\mathrm{III}_{x-y}$ are both reentrant. A reentrant tongue of Phase $\mathrm{IV}_{x}$ at low drives appears for $A / B>0.55$. At all values of $A / B$, the flow at large $f_{D C}$ is strictly in the $x$ direction (Phase $\operatorname{IV}_{x}$ ). The width of the pinned Phase $\mathrm{I}_{p}^{*}$ increases upon approaching $A / B=1$ from below since the almost circular particle orbit around one potential maximum is highly stable. Motion in the $y$ direction $\left(\right.$ Phase $\left.\mathrm{II}_{y}\right)$ still appears for the symmetric ac drive $A / B=1$ due to the fact that the particular chirality of the ac drive breaks the reflection symmetry across the $y$ axis.

For $A / B>0.6$, the sliver of reentrant Phase III $_{x-y}$ falling between Phases $\mathrm{II}_{y}$ and $\mathrm{IV}_{x}$ becomes gradually smaller as $A / B$ increases until it vanishes above $A / B=$ 0.88. The transition from Phase $\mathrm{II}_{y}$ to Phase $\mathrm{IV}_{x}$ at higher values of $A / B>0.88$ occurs in a very small window of of $f_{D C}$ but is not completely sharp. Instead there is a small region where the flowing particle moves in both the $x$ and $y$ direction but at an angle less than $45^{\circ}$. The flow is intermittent and jumps among different orbits or angles. We have also considered the effect of starting the particle at a nonzero fixed $f_{D C}$ in the transition region between Phases $\mathrm{II}_{y}$ and $\mathrm{IV}_{x}$ and find that the flow will settle quickly to Phase $\mathrm{II}_{y}$ or $\mathrm{IV}_{x}$.

We now consider the conditions under which transverse mobility and the reentrant pinning can occur, and indicate where the boundaries between the different phases are expected to fall.

Pinned phases $I_{p}$ and $I_{p}^{*}$ : The particle remains in the pinned phase $\mathrm{I}_{p}$ as long as the combined dc and maximum ac components are less than the confining barrier produced by the repulsive obstacles. This barrier has a strong angular dependence due to its egg-carton shape, and the lowest points of the barrier fall at the center of each of the four sides of the plaquette, at $x_{\min }^{ \pm}$and $y_{\min }^{ \pm}$, where the $\mathrm{x}$ - or $\mathrm{y}$-confining forces pass through a minimum. The largest thresholds occur for a particle trajectory along a $45^{\circ}$ angle passing through the potential maximum. At $A / B \approx 0.88$, a transition to a new pinned phase $\mathrm{I}_{p}^{*}$ occurs. For $A / B<0.88$, the pinned particle orbit is contained inside a single plaquette. For $A / B>0.88$, the orbit becomes too large to fit inside the plaquette, and the particle switches to a larger orbit centered around one of the potential maxima.

Transition from $I_{p}$ to $I I_{y}$ : For $A=0$ and fixed $\mathrm{B}$, the particle orbit consists of a single line extending from the top to the bottom of the plaquette, close to the minimum y-confining force points of the potential, $y_{\min }^{ \pm}$. Due to this proximity induced by the y-component of the ac drive, the particle depins in the y-direction before it depins in the $\mathrm{x}$-direction for nonzero values of $\mathrm{A}$, and enters phase $\mathrm{II}_{y}$. The particle hops from one plaquette to the next plaquette in the positive y-direction during a brief interval at the end of the rising phase of the particle orbit. In order to make this hop, the particle must be moving rapidly enough to reach the next plaquette before the ac phase reaches the downward portion of the cycle. Increasing $f_{D C}$ or increasing $A$ both contribute to increasing the velocity of the particle during the hop. The minimum $f_{D C}$ value required to permit the particle to hop at low but nonzero $A$ is $f_{D C} \approx 0.11$. As $A / B$ increases, the $\mathrm{x}$-component of the ac force also contributes to the particle velocity during the hop, so the value of $f_{D C}$ that must be applied to induce y-direction motion drops, such that the particle velocity during the hop at the onset of phase $\mathrm{II}_{y}$ remains roughly constant as $A / B$ increases.

Transition from $I I_{y}$ to $I_{p}$ : As $f_{d}$ increases further within Phase $\mathrm{II}_{y}$, for $A / B<0.45$, a transition to a reentrant pinned phase occurs. Here, the x-velocity of the particle has increased enough that the particle is swept past the minimum in the y-confining potential, $y_{\min }^{+}$, before it has time to hop to the next plaquette. Thus the particle returns to a pinned orbit. The $\mathrm{II}_{y}-\mathrm{I}_{p}$ transition line moves to higher $f_{d}$ with increasing $A / B$ due to the fact that the time required for the particle to complete its hop to the next plaquette drops as $A / B$ increases. Therefore, a higher $f_{D C}$ is required to sweep the particle past $y_{\min }^{+}$before the hop is complete.

Transition from $I_{p}$ to $I V_{x}$ : Beyond the second pinned phase $\mathrm{I}_{p}$, as $f_{D C}$ is further increased, the particle orbit is shifted closer to the minimum in the x-confining potential on the right side of the plaquette, $x_{\min }^{+}$, and when the combined ac and dc forces in the $\mathrm{x}$-direction exceed the potential strength at this minimum, the particle depins in the positive x-direction. For $A / B<0.45, f_{D C}$ at the depinning transition decreases with increasing $A / B$ due to the fact that the ac force also contributes to the net $\mathrm{x}$-force on the particle. This contribution saturates at $A / B \approx 0.45$, when the $\mathrm{II}_{y}-\mathrm{I}_{p}$ line meets the $\mathrm{I}_{p}-\mathrm{IV}_{x}$ line and the reentrant phase disappears.

Transition from $I I_{y}$ to $I V_{x}$, with a reentrant $I I I_{x-y}$ : For $A / B>0.45$, as $f_{D C}$ is increased, the particle motion leaves Phase $\mathrm{II}_{y}$, passing briefly through a sliver of Phase $\mathrm{III}_{x-y}$ before entering phase $\mathrm{IV}_{x}$, with motion in the x-direction only. Depinning of the particle in the $\mathrm{x}$-direction first occurs when the x-component of the ac drive combined with $f_{D C}$ exceeds the x-confining force of the potential at $x_{\min }^{+}$. At this value of $f_{D C}$, the particle is moving in both the $\mathrm{x}$ and $\mathrm{y}$ directions in phase $\mathrm{III}_{x-y}$. Due to the $\mathrm{x}$-motion of the particle, however, the $\mathrm{y}$-motion becomes unstable, and at slightly higher values of $f_{D C}$, the y-motion ends and the particle moves only in the $\mathrm{x}$-direction in phase $\mathrm{IV}_{x}$. As the drive is further increased, stable y-motion becomes possible again, and the particle enters a wide region of phase $\mathrm{III}_{x-y}$.

Transition from $I I I_{x-y}$ to $I V_{x}$ at high $f_{D C}$ : As $f_{D C}$ is increased further, the y-direction motion of phase $\mathrm{III}_{x-y}$ 


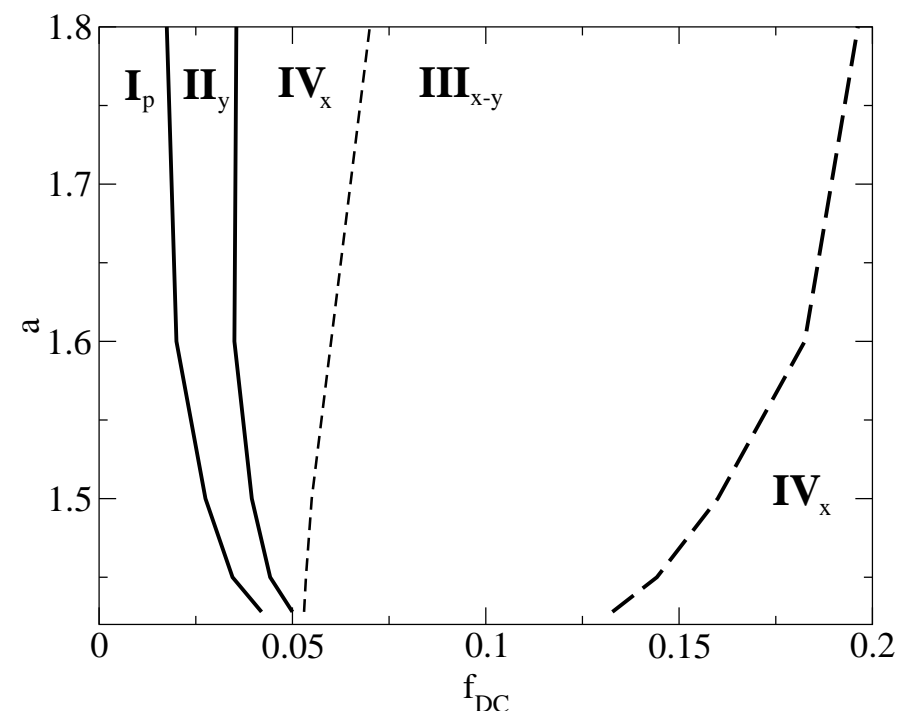

FIG. 4. Dynamic phase diagram for substrate lattice constant $a$ vs $f_{D C}$ for a system with $A=B$ and $\omega_{A}=\omega_{B}$. Phase $\mathrm{I}_{p}$ : pinned phase. Phase $\mathrm{II}_{y}$ : motion only in the $y$-direction. Phase $\mathrm{III}_{x-y}$ : motion at $45^{\circ}$. Phase $\mathrm{IV}_{x}$ : motion only the in $x$-direction.

ends when the particle orbit becomes so extended in the $\mathrm{x}$-direction due to the increased $f_{D C}$ that it can no longer depin in the y-direction since it has moved away from the $\mathrm{y}$-minimum location $y_{\min }^{+}$.

We next consider the effect of changing the density of the system. We achieve this by performing a series of simulations for fixed $A / B=1.0$ while varying the lattice constant of the periodic substrate and changing the system size accordingly. This increases the effective substrate strength since it increases the barrier to hop from one plaquette to another. In Fig. 4 we show the phase diagram of the substrate lattice constant $a$ vs $f_{D C}$. We consider lattice constants ranging from $1.42 \lambda$ to $1.8 \lambda$. New dynamic phases appear for lattice constants outside of this range. For smaller lattice constants $a<1.42$, the zero-dc drive orbits start to encircle two or more potential maxima. For larger lattice constants, $a>1.8$, the phases start to show disordered or chaotic behavior, and Phases $\mathrm{I}_{p}$ to $\mathrm{IV}_{x}$ become difficult to define. Fig. 4 shows that the width of the pinned Phase $\mathrm{I}_{p}$ grows for denser systems, as expected due to the increased barriers for inter-plaquette jumps. It might be expected that Phase $\mathrm{II}_{y}$ would grow for smaller $a$ as the plaquettes shrink; however, the increased repulsion caused by the shorter distance between the potential maxima and the particle causes the particle orbit to shrink as well. Both Phase $\mathrm{II}_{y}$ and the first Phase $\mathrm{IV}_{x}$ shrink with decreasing $a$, while the second onset of Phase $\mathrm{IV}_{x}$ occurs at a lower value of $f_{D C}$ since the particle does not need to move as far in the $\mathrm{x}$-direction in order to reach the next plaquette.

We have also considered the effects of adding a phase shift to the ac drives. We shift the phase of the $\mathrm{x}-$ component of the ac drive by $\delta: A \sin \left(\omega_{A} t+\delta\right) \hat{\mathbf{x}}$. This

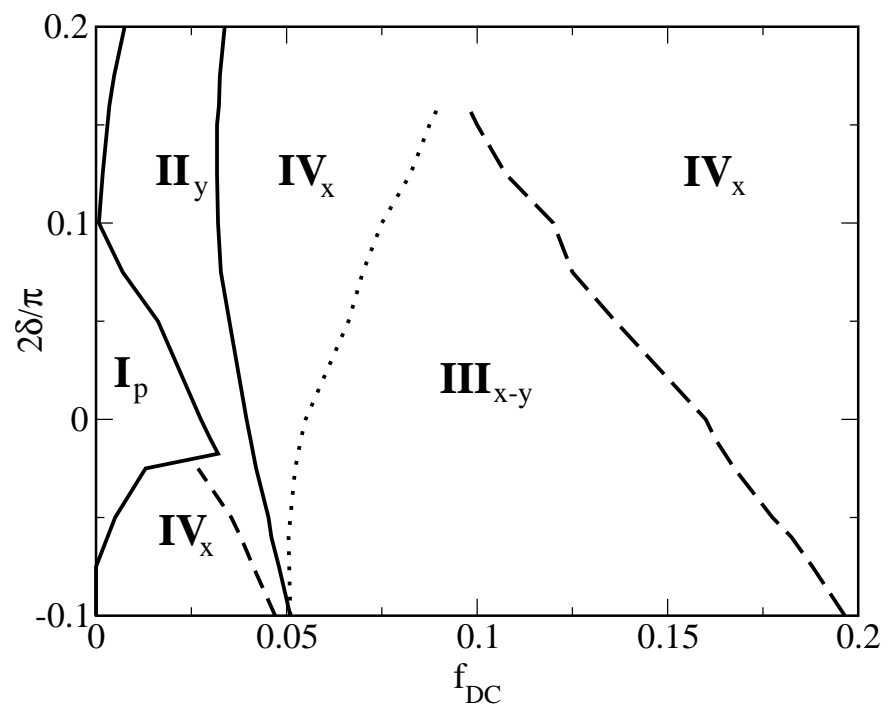

FIG. 5. Dynamic phase diagram for the phase shift $\delta$ in units of $2 / \pi$ vs $f_{D C}$ for a system with $\omega_{A}=\omega_{B}$ and $A=B$. The phase shift is added to the $x$-component of the ac drive. Phase $\mathrm{I}_{p}$ : pinned phase. Phase $\mathrm{II}_{y}$ : motion only in the $y$-direction. Phase $\mathrm{III}_{x-y}$ : motion at $45^{\circ}$. Phase $\mathrm{IV}_{x}$ : motion only the in $x$-direction.

causes the particle orbits to become tilted and elliptical. We restrict ourselves to the region $-0.05 / \pi<\delta<0.1 / \pi$ in phase space where the four phases described above occur. For larger shifts new phases can arise. In Fig. 5 we plot the phase diagram as a function of phase shift $\delta$ vs $f_{D C}$. At $\delta=\pi / 2$ the ac orbit would be a straight line along $45^{\circ}$. Fig. 5 shows that as $\delta$ increases from zero, which corresponds to the elliptical orbit being tilted toward the right, Phase $\mathrm{II}_{y}$ increases in size and Phase $\mathrm{I}_{p}$ decreases in size. For large enough shifts $\delta$, Phase $\mathrm{III}_{x-y}$ disappears. For negative phase shifts a new region of Phase $\mathrm{IV}_{x}$ appears between Phases $\mathrm{I}_{p}$ and $\mathrm{II}_{y}$. For large enough negative phase shifts we observe a ratchet effect in which the particle moves in the $x$-direction with zero dc drive. We discuss this more in the next section. Also for increasing negative phase shifts, $\mathrm{Phase}_{\mathrm{II}}$ decreases in size while Phase $\mathrm{III}_{x-y}$ increases in size.

\section{RATCHET EFFECTS}

The elliptical ac drives in the previous sections preserve the combined $x$ and $y$ reflection symmetries of the system. ac drives with additional asymmetries can produce a net dc motion or ratchet effect in the absence of a dc drive. We consider a system with $f_{D C}=0$ under an asymmetric applied ac drive of the form

$$
\mathbf{f}_{A C}=A\left[\sin \left(\omega_{A} t\right)+\sin ^{3}\left(\omega_{B} t\right)\right] \hat{\mathbf{x}}-B \cos \left(\omega_{B} t\right) \hat{\mathbf{y}}
$$

where $\omega_{B} / \omega_{A}=0.8$. In Fig. 6 (a) we plot $V_{y}$ vs $A$ for constant $B=0.34$ and $f_{D C}=0$. In this regime, $V_{x}=0$. There are a series of regions for increasing $A$ that have 

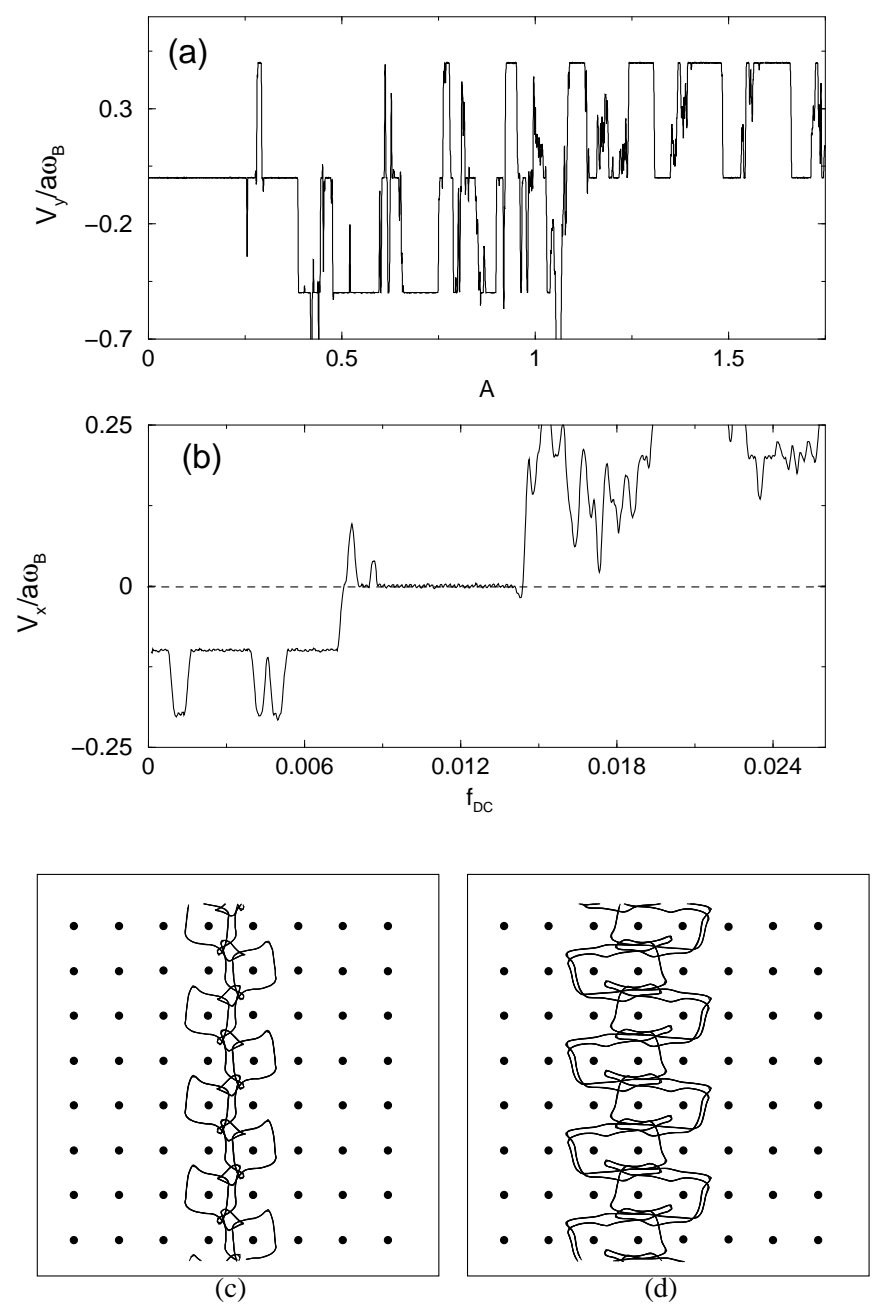

FIG. 6. (a) Transverse velocity $V_{y} / a \omega_{B}$ vs $A$ at $f_{D C}=0$ and $B=0.34$ for a system with an asymmetric ac drive, $\omega_{B} / \omega_{A}=0.8$. (b) Longitudinal velocity $V_{x}$ vs $f_{D C}$ for a system at $B / A=0.7$ showing a negative ratchet effect at $f_{D C}<0.0075$. (c) Particle trajectory for a positive $V_{y}$ orbit from panel (a) at $A=0.285, B=0.34$, and $f_{D C}=0$. (d) Particle trajectory for a negative $V_{y}$ orbit from panel (a) at $A=0.5, B=0.34$, and $f_{D C}=0$.

a finite dc value of $V_{y}$ in both the positive and negative direction, indicating a ratchet effect with velocity $a \omega_{B} / 2$. In Fig. 6(c) we illustrate a positive $V_{y}$ orbit from the system in Fig. 6(a) at $A=0.285$. The orbit has alternating lobes which are angled in the positive $y$ direction. In Fig. $6(\mathrm{~d})$ we show that the negative $V_{y}$ orbit at $A=0.5$ is composed of an alternating double rectangular orbit which is tilted in the negative $y$ direction.

We have also found ratchet regimes where there is a finite dc velocity in the opposite direction of the applied dc drive. In Fig. 6(b), we plot $V_{x}$ vs $f_{D C}$ for a system with $\mathbf{f}_{A C}=A \sin \left(\omega_{A} t\right) \hat{\mathbf{x}}-B \cos \left(\omega_{B} t\right) \hat{\mathbf{y}}, B / A=0.7$, and $\omega_{A} / \omega_{B}=1.85$. Here there is a regime $0<f_{D C}<0.0075$ where the particle is moving backward with respect to the dc drive, which is applied in the positive $x$-direction.
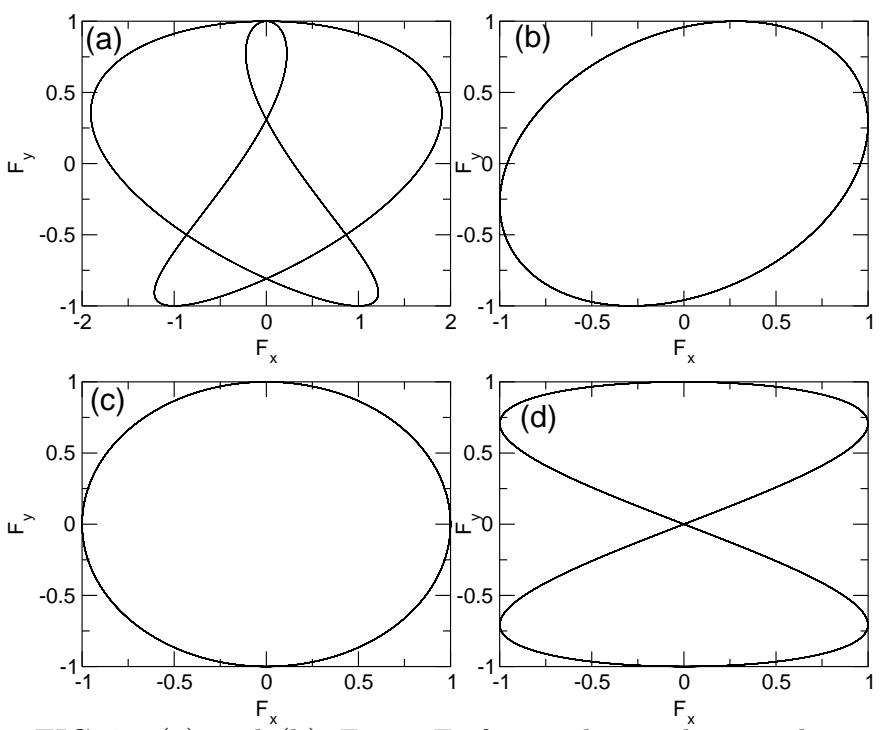

FIG. 7. (a) and (b) $F_{x}$ vs $F_{y}$ for ac drives that produce a ratchet effect. (a) $\mathbf{f}_{A C}=A \sin \left(\omega_{A} t\right) \hat{\mathbf{x}}+A \sin \left(1.5 \omega_{A} t\right) \hat{\mathbf{x}}-B \cos \left(1.5 \omega_{B} t\right) \hat{\mathbf{y}}$, $A / B=1, A=1$, and $\omega_{A} / \omega_{B}=1$. (b) A ratchet effect produced by a phase shift: $\mathbf{f}_{A C}=A \sin \left(\omega_{A} t+\delta\right) \hat{\mathbf{x}}-B \cos \left(\omega_{B} t\right)$, $\delta=0.287, A / B=1$, and $\omega_{A} / \omega_{B}=1$. (c) and (d) $F_{x}$ vs $F_{y}$ for ac drives that do not produce a ratchet effect. (c) $\mathbf{f}_{A C}=A \sin \left(\omega_{A} t\right) \hat{\mathbf{x}}-B \cos \left(\omega_{B} t\right) \hat{\mathbf{y}}$ with $A / B=1$ and $\omega_{A} / \omega_{B}=1$. (d) $\mathbf{f}_{A C}=A \sin \left(\omega_{A} t\right) \hat{\mathbf{x}}-B \cos \left(2 \omega_{B} t\right) \hat{\mathbf{y}}$ with $A / B=1$ and $\omega_{A} / \omega_{B}=1$.

The system enters a pinned phase for $0.009<f_{D C}<$ 0.014 before beginning to move strictly in the positive $x$-direction for $f_{D C}>0.014$. We note that this negative velocity in opposition to the dc drive is not a negative mobility regime. Instead it is a ratchet effect which can persist for a range of opposite dc drive. The fact that there is a finite negative velocity even at $f_{D C}=0.0$ as seen in Fig. 6(b) shows that the dc drive is not causing the net dc motion.

A basic question is what are the minimal ac drive criteria required to produce a ratchet effect at $f_{D C}=0.0$. In general we find that ratchet effects occur for ac drives in which at least one of the spatial reflection symmetries is broken. An example of a simple ac drive that produces a zero-dc ratchet effect is $\mathbf{f}_{A C}=A \sin \left(\omega_{A} t\right) \hat{\mathbf{x}}+$ $A \sin \left(1.5 \omega_{A} t\right) \hat{\mathbf{x}}-B \cos \left(1.5 \omega_{B} t\right) \hat{\mathbf{y}}$, with $A / B=1$ and $\omega_{A} / \omega_{B}=1$. In Fig. $7(\mathrm{a})$ we plot $F_{x}$ vs $F_{y}$ for this ac drive in the absence of a substrate with $A=1.0$. In Fig. 8(a) we show the particle motion over the substrate for $f_{D C}=0.0$. The particle translates in the negative $y$-direction. Fig. 7(a) shows that the ac drive breaks a spatial symmetry across the $y$-axis. The orbit of the particle thus breaks an $x$-axis symmetry. In Fig. $7(\mathrm{c})$ we plot $F_{x}$ vs $F_{y}$ for $\mathbf{f}_{A C}=A \sin \left(\omega_{A} t\right) \hat{\mathbf{x}}-B \cos \left(\omega_{B} t\right) \hat{\mathbf{y}}$ with $A / B=1$ and $\omega_{A} / \omega_{B}=1$, and in Fig. $7(\mathrm{~d})$ we plot $F_{x}$ vs $F_{y}$ for $\mathbf{f}_{A C}=A \sin \left(\omega_{A} t\right) \hat{\mathbf{x}}-B \cos \left(2 \omega_{B} t\right) \hat{\mathbf{y}}$ with $A / B=1$ and $\omega_{A} / \omega_{B}=1$. These drives do not produce a zero-dc ratchet effect, and these orbits do not break 


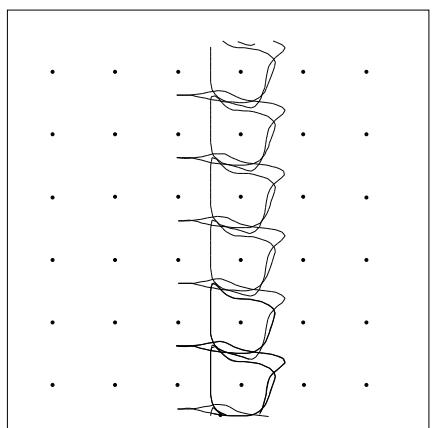

(a)

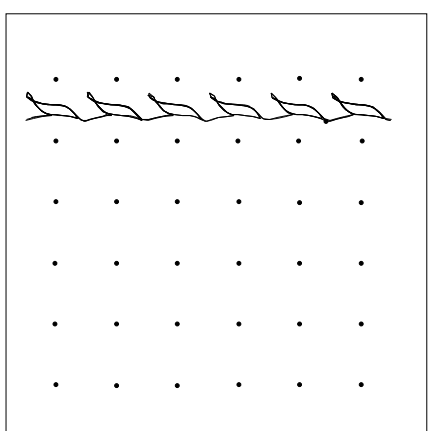

(b)
FIG. 8. The particle trajectories (black line) for a fixed time interval and $f_{D C}=0.0$ for motion in a $2 \mathrm{D}$ periodic substrate with potential maxima located at the black dots. (a) A ratchet effect due to the breaking of a reflection symmetry. The particle moves in the negative y-direction when driven with the ac drive shown in Fig. 7(a), $\mathbf{f}_{A C}=A \sin \left(\omega_{A} t\right) \hat{\mathbf{x}}+A \sin \left(1.5 \omega_{A} t\right) \hat{\mathbf{x}}-B \cos \left(1.5 \omega_{B} t\right) \hat{\mathbf{y}}$, $A / B=1, \omega_{A} / \omega_{B}=1$. (b) A ratchet effect produced by the addition of a phase shift. The particle moves in the positive $\mathrm{x}$-direction when driven with the ac drive shown in Fig. 7(b), $\mathbf{f}_{A C}=A \sin \left(\omega_{A} t+\delta\right) \hat{\mathbf{x}}-B \cos \left(\omega_{B} t\right), \delta=0.287, A / B=1$, and $\omega_{A} / \omega_{B}=1$.

a reflection symmetry. As indicated in the phase diagram of Fig. 6, the addition of a phase shift to the ac drive in the $\mathrm{x}$-direction produces another simple ac drive that exhibits a zero-dc ratchet effect. In Fig. 8(b) we show a particle orbit under the addition of a negative phase shift, $\mathbf{f}_{A C}=A \sin \left(\omega_{A} t+\delta\right) \hat{\mathbf{x}}-B \cos \left(\omega_{B} t\right)$ with $\delta=0.287, A / B=1$, and $\omega_{A} / \omega_{B}=1$, that produces a zero-dc ratchet effect in the x-direction. $F_{x}$ vs. $F_{y}$ for this orbit is illustrated in Fig. 7(b). The ac drive in Fig. $7(\mathrm{~b})$ breaks both the $x$ and $y$ reflection symmetries. We note that even if an ac drive breaks a reflection symmetry it does not necessarily produce a zero-dc ratchet. However, we have never observed zero-dc ratcheting for symmetrical ac drives.

\section{SUMMARY}

We have presented a simple model of a particle driven in a two-dimensional symmetric periodic substrate with an elliptical ac drive. In certain regimes, the particle moves strictly in the $y$-direction when the dc drive is applied in the $x$-direction. We term this effect absolute transverse mobility. Additionally we have mapped several dynamic phase diagrams for this system, which feature a number of dynamical phases including simultaneous motion in the $x$ and $y$ direction, as well as a reentrant pinned phase. For ac drives which produce asymmetric orbits, net dc motion or a zero-dc ratchet effect can arise in the absence of an applied dc drive. Our results can be tested experimentally and have useful applications for controlling flux motion in superconductors, colloids in optical trap arrays, and biomolecules moving through a periodic obstacle array.

We thank C. Bechinger, D. Grier, M.B. Hastings, and P. Korda for useful discussions. This work was supported by the US DOE under Contract No. W-7405-ENG-36.

[1] G. Grüner, Rev. Mod. Phys. 60, 1129 (1988).

[2] G. Blatter, M.V. Feigel'man, V.B. Geshkenbein, A.I. Larkin, and V.M. Vinokur, Rev. Mod. Phys. 66, 1125 (1994).

[3] G.A. Cecchi and M.O. Magnasco, Phys. Rev. Lett. 76, 1968 (1996).

[4] C. Reichhardt, C.J. Olson and F. Nori, Phys. Rev. B 58, 6534 (998).

[5] M.O. Magnasco, Phys. Rev. Lett. 71, 1477 (1993); R.D. Astumian and M. Bier, ibid. 72, 1766 (1994); C.R. Doering, W. Horsthemke, and J. Riordan, ibid. 72, 2984 (1994); P. Reimann, Phys. Rep. 361, 57 (2002).

[6] R. Bartussek, P. Hänggi, and J.C. Kissner, Europhys. Lett. 28, 459 (1994); J.L. Mateos, Phys. Rev. Lett. 84, 258 (2000).

[7] P. Reimann, R. Kawai, C. van den Broeck, and P. Hänggi, Europhys. Lett. 45, 545 (1999); J. Buceta, J.M. Parrondo, C. Van den Broeck, and F.J. de la Rubia, Phys. Rev. E 61, 6287 (2000); B. Cleuren and C. Van den Broeck, Europhys. Lett. 54, 1 (2001).

[8] R. Eichhorn, P. Reimann, and P. Hänggi, Phys. Rev. Lett. 88, 190601 (2002); Phys. Rev. E. 66, 066132 (2002).

[9] K. Seeger, Semiconductor Physics (Springer-Verlag, Berlin, 1982); E. Scholl, Nonequilibrium Phase Transitions in Semiconductors (Springer-Verlag, Berlin, 1987); S. Wang and D.D.L. Chung, Composites B, 30, 579 (1999).

[10] C. Reichhardt, C.J. Olson and M.B. Hastings, Phys. Rev. Lett. 89, 024101 (2002).

[11] M. Baert, V.V. Metlushko, R. Jonckheere, V.V. Moshchalkov, and Y. Bruynseraede, Phys. Rev. Lett. 74, 3269 (1995); S.B. Field, S.S. James, J. Barentine, V. Metlushko, G. Crabtree, H. Shtrikman, B. Ilic, and S.R.J. Brueck, Phys. Rev. Lett. 88, 067003 (2002).

[12] K. Harada, O. Kamimura, H. Kasai, T. Matsuda, A. Tonomura, and V.V. Moshchalkov, Science 274, 1167 (1996).

[13] L. Van Look, E. Rosseel, M.J. Van Bael, K. Temst, V.V. Moshchalkov, and Y. Bruynseraede, Phys. Rev. B 60, R6998 (1999).

[14] J.I. Martín, M. Vélez, A. Hoffmann, I.K. Schuller, and J.L. Vicent, Phys. Rev. Lett. 83, 1022 (1999).

[15] C. Reichhardt, A.B. Kolton, D. Domínguez, and N. Grønbech-Jensen, Phys. Rev. B 64, 134508 (2001).

[16] P.T. Korda, M.B. Taylor, and D.G. Grier, Phys. Rev. Lett. 89, 128301 (2002).

[17] M Brunner and C. Bechinger, Phys. Rev. Lett. 88, 248302 (2002); K. Man- 
gold, P. Leiderer, and C. Bechinger, Phys. Rev. Lett. 90, 158302 (2003).

[18] J.E. Curtis, B.A. Koss, and D.G. Grier, Opt. Comm. 207, 169 (2002).

[19] W.D. Volkmuth and R.H. Austin, Nature (London) 358, 600 (1992); C.-F. Chou, O. Bakajin, S.W.P. Turner, T.A.J. Duke, S.S. Chan, E.C. Cox, H.G. Craighead, and R.H. Austin, Proc. Natl. Acad. Sci. U.S.A. 96, 13762 (1999); J.-L. Vioy, Rev. Mod. Phys. 72, 813 (2000).

[20] D. Weiss, M.L. Roukes, A. Menschig, P. Grambow, K. von Klitzing, and G. Weimann, Phys. Rev. Lett. 66, 2790 (1991); G.R. Nash, S.J. Bending, M. Riek, and K. Eberl, Phys. Rev. B 63, 113316 (2001); W. Breuer, D. Weiss, and V. Umansky, Physica E 12, 216 (2002).

[21] G. Grynberg and C. Robilliard, Phys. Rep. 56, 335 (2001); M. Schiavoni, F.-R. Carminati, L. Sanchez-Palencia, F. Renzoni, and G. Grynberg, Europhys. Lett. 59, 493 (2002).

[22] N. Grønbech-Jensen, Int. J. Mod. Phys. C 7, 873 (1996). 